ISSN 0103-5150

Fisioter. Mov., Curitiba, v. 24, n. 3, p. 513-522, jul./set. 2011

Licenciado sob uma Licença Creative Commons

\title{
Evolução científica da fisioterapia em 40 anos de profissão
}

\author{
The scientific evolution of the Brazilian physical therapy in \\ 40 years as a profession
}

\section{Cristiane de Carvalho Lima Calvalcante ${ }^{[a]}$, Ana Rosa de Sousa Rodrigues ${ }^{[b]}$, Thais Varanda Dadalto ${ }^{[\mathrm{c}]}$, Elirez Bezerra da Silva ${ }^{[\mathrm{d}]}$}

[a] Graduada em Fisioterapia, Mestranda do Programa de Pós-Graduação Stricto Sensu em Educação Física, Universidade Gama Filho (UGF), Rio de Janeiro, Rio de Janeiro - Brasil, e-mail: crisdclima@hotmail.com

[b] Graduada em Fisioterapia, Especialista em Acupuntura, professora do curso de Graduação em Fisioterapia (UGF), Mestranda do Programa de Pós-Graduação Stricto Sensu em Educação Física, Universidade Gama Filho (UGF), Rio de Janeiro, Rio de Janeiro - Brasil.

[c] Graduada em Fisioterapia, Mestranda do Programa de Pós-Graduação Stricto Sensu em Educação Física, Universidade Gama Filho (UGF), Rio de Janeiro, Rio de Janeiro - Brasil.

[d] Graduado em Fisioterapia e Educação Física, Mestre e Doutor em Educação Física, coordenador do curso de Graduação em Fisioterapia, Universidade Gama Filho (UGF), professor do Programa de Pós-Graduação Stricto Sensu em Educação Física, Universidade Gama Filho (UGF), Rio de Janeiro, Rio de Janeiro - Brasil, e-mail: elirezsilva@ugf.br

\section{Resumo}

Introdução: Em 13 de outubro de 1969 com o Decreto-Lei 938, a fisioterapia se legitimou como profissão e desde então vem evoluindo a prática clínica com a pesquisa científica. Objetivos: 0 presente estudo teve por objetivo levantar a evolução científica da fisioterapia brasileira em 40 anos de profissão. Materiais e métodos: Foram realizadas buscas em bases de dados eletrônicas MEDLINE e Biblioteca Virtual de Saúde, nos sites SCImago, Google Acadêmico, Capes, CNPq e Inep, com filtro para título e palavras-chave na língua inglesa e portuguesa: fisioterapia, evidências científicas e área da saúde. Resultados: Somente há 40 anos foi estabelecido que os fisioterapeutas diplomados por escolas e cursos reconhecidos são profissionais de nível superior. Há 1.145 fisioterapeutas/TO com curso de doutorado e 4.675 com curso de mestrado. Isto representa 4\% e 11\%, respectivamente, dos profissionais stricto sensu da grande área da saúde. Dentro das profissões de saúde, a fisioterapia ocupa a penúltima posição em quantidade de doutores. A produção científica da Fisioterapia brasileira em relação ao mundo ocupa, de acordo com o ranking do SCImago, de 1996 a 


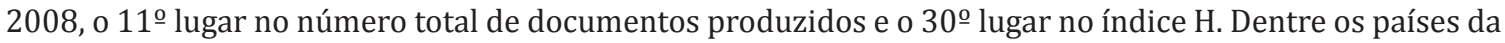
América Latina, ocupa o $1^{\text {o }}$ lugar na maioria dos quesitos. Conclusão: Ainda há muito para se fazer a fim de aumentar a quantidade de pesquisadores, com a criação de outros programas stricto sensu.

Palavras-chave: Ciência. Formação. Educação. Fisioterapia.

\begin{abstract}
Introduction: On October 13, 1969 the physiotherapy was legitimized throughthe Decree-Law 938 and has been evolving clinical practice with science research. Objectives: The present study aimed at assessing the scientific evolution of Physiotherapy in Brazil 40 years of occupation. Materials and methods: We searched in electronic databases MEDLINE and Health Virtual Library, SCImago sites, Google Scholar, Capes, CNPq and Inep, with filter for title and keywords in English and Portuguese Physical Therapy, scientific evidence and health. Results: Only 40 years ago it was established that the physiotherapist graduates of schools and courses are recognized top-level professionals. There are 1145 physiotherapists/TO with graduate school and 4,675 with the masters course. This represents $4 \%$ and $11 \%$ respectively, strictly speaking professionals from the health field. Within the health professions, physiotherapy occupies the penultimate position in number of doctors. The Brazilian scientific production in Physiotherapy from the world stands, according to the ranking SCImago 1996 to 2008, the 11th in the total number of documents produced and the 30th place in the index $H$. Among Latin American countries, occupies the first place on most items. Conclusion: There is still much to do in order to increase the number of researchers, with the creation of other graduate studies program.
\end{abstract}

Keywords: Science. Formation. Education. Physiotherapy.

\section{Introdução}

A fisioterapia, uma das áreas mais jovens da saúde, nasceu e vem se desenvolvendo no Brasil por meio dos esforços de uma categoria que, até 2004, era de aproximadamente 80 mil profissionais (1).

Se considerarmos a definição sociológica/antropológica de uma profissão, parece que a sua existência depende da ciência, dos conhecimentos científicos produzidos nessa profissão. Para Wilensky (2), qualquer profissão que queira exercer sua autoridade profissional deve ter um amparo jurídico, baseado nas leis, portarias, pareceres e resoluções e deve também convencer a sociedade de que seus serviços são confiáveis. Latour (3) considera também muito mais importante para a profissão a eleição de poderosas estratégias para convencimento dos seus próprios pares, da sociedade e do Estado quanto à sua utilidade, necessidade de sua existência, e até mesmo, necessidade de sua continuidade. A principal forma de convencer a sociedade que seus serviços são confiáveis, necessários, úteis, importantes e que, por isso mesmo, devem ser mantidos é dando sustentação científica às intervenções praticadas pela profissão. A sustentação científica é dada pelo conhecimento gerado nos institutos de pesquisas e programas de mestrado e doutorado, difundido nas revistas especializadas.

0 presente estudo teve como objetivo levantar a evolução científica da fisioterapia brasileira em 40 anos de profissão.

\section{Estratégia de busca}

Foram realizadas buscas em bases de dados eletrônicas, como MEDLINE e Biblioteca Virtual de Saúde, nos sites SCImago, Google Acadêmico, Capes, CNPq e Inep, com filtro para título e palavras-chave na língua inglesa e portuguesa: fisioterapia, evidências científicas e área da saúde.

\section{História da fisioterapia}

A história da profissão de fisioterapeuta no País tem sido objeto de estudo de professores e pesquisadores, principalmente fisioterapeutas vinculados a programas de pós-graduação em áreas como saúde coletiva, educação e na própria fisioterapia (4). 
A fisioterapia no Brasil se confunde um pouco com a história da poliomielite e com o surgimento de recursos para tratar as sequelas dessa doença. A Escola de Reabilitação do Rio de Janeiro foi criada pela Associação Brasileira Beneficente de Reabilitação (ABBR), em 1956, e foi a primeira instituição no País a oferecer em caráter regular um curso de graduação em Fisioterapia (5).

A luta de um grupo de profissionais fez crescer a profissão no aspecto legal. Com o surgimento do Parecer 388/63, elaborado por uma comissão de peritos, no Conselho Federal de Educação, e aprovado em 10 de dezembro de 1963 pelo Ministério de Educação e Cultura (MEC), foram reconhecidos os cursos de Fisioterapia. Tal parecer definia que esses cursos deveriam ter a duração de três anos e estabelecia um currículo mínimo, caracterizando pela primeira vez os profissionais aqui chamados de Técnicos em Fisioterapia, cujas funções também foram definidas nesse Parecer (6).

Contudo, somente em 13 de outubro de 1969, com o Decreto-Lei 938, a fisioterapia se legitimou como profissão. 0 art. $2^{\circ}$ definiu que os fisioterapeutas diplomados por escolas e cursos reconhecidos são profissionais de nível superior e o art. $3^{\circ}$ definiu como sendo atividade privativa do fisioterapeuta executar métodos e técnicas fisioterapêuticas com a finalidade de restaurar, desenvolver e conservar a capacidade física do paciente (6).

Diversos trabalhos têm abordado períodos recentes da profissionalização da fisioterapia, tendo o Decreto-lei n. 938 como ponto de partida de suas análises, passando pela criação dos Conselhos de Fisioterapia e de Terapia Ocupacional, nos anos 1970, e utilizando, por vezes, a história como pano de fundo para suas produções acadêmicas (7).

A Fisioterapia apresentou mudanças no decorrer dos 40 anos legais de profissão. No início, a profissão se baseava em livros de reabilitação e algumas técnicas, como Bobath, Kabat, etc. Felizmente, essa tendência sofreu grandes mudanças. Hoje a prática clínica é necessariamente alicerçada em pesquisa e nos seus resultados, confirmando cada vez mais um interesse do fisioterapeuta na prática baseada em evidências (8).

\section{Fisioterapia stricto sensu}

No dia 20 de dezembro de 1996, o MEC, por meio da Coordenação de Aperfeiçoamento de Pessoal de
Nível Superior (Capes), autorizou oficialmente o funcionamento do primeiro mestrado brasileiro em Fisioterapia na Universidade de São Carlos (UFSCar). A partir disso, a fisioterapia passou a integrar formalmente a comunidade científica brasileira (9).

A criação do primeiro mestrado em Fisioterapia facilitou a qualificação stricto sensu dos fisioterapeutas na própria área, que antes era feita no exterior ou aqui mesmo no Brasil, mas em áreas afins, como anatomia, educação, morfologia, psicologia e outras semelhantes (9).

A partir daí, além da abertura de outros cursos stricto sensu na área (10), outros fatos de igual relevância ocorreram na busca da solidificação da fisioterapia junto à comunidade científica da grande área de ciências da saúde. Entre elas, podemos citar a criação da Associação Brasileira de Pesquisa e Pós-Graduação em Fisioterapia (ABRAPG-Ft), o surgimento de Fóruns Nacionais de Pesquisa e PósGraduação Stricto Sensu em Fisioterapia (11) e a representação formal da área de fisioterapia e terapia ocupacional no Conselho Nacional de Desenvolvimento Científico e Tecnológico (CNPq) (12), o que criou um espaço para discussão da pós-graduação stricto sensu e pesquisa em fisioterapia no Brasil e abriu um canal para a articulação política da classe junto aos órgãos de fomento à pesquisa e aos de divulgação da produção científica (13).

0 mestrado é um curso que tem duração média de dois anos e pode ser de dois tipos: o mestrado acadêmico ou científico e o profissional. Segundo o Parecer 79/2002 do CNE/CES, esses dois tipos de mestrado conferem grau e prerrogativas idênticas, inclusive para o exercício da docência e, como todo programa de pós-graduação stricto sensu, têm a validade nacional do diploma condicionada ao reconhecimento do curso. Ou seja, o diploma de um mestrado acadêmico vale o mesmo que um diploma de mestrado profissional (14). Ainda se referindo aos cursos de pós-graduação stricto sensu, o doutorado é um curso que tem duração média de quatro anos e somente pode ser acadêmico.

Atualmente, existem dentro da grande área de ciências da saúde 303 cursos de doutorado, 412 de mestrado acadêmico e 45 de mestrado profissional (Tabela 1) (15). Dentro das ciências da saúde, existem 11 áreas de avaliação: educação física, enfermagem, farmácia, fisioterapia e terapia ocupacional, fonoaudiologia, medicina I, II e III, odontologia, nutrição e saúde coletiva. Comparando a fisioterapia e a terapia 
ocupacional com as outras áreas de avaliação das ciências da saúde, em números de mestrados acadêmicos a fisioterapia ocupa a $10^{\mathrm{a}}$ posição e de doutorados, a última posição. Não existem mestrados profissionais em fisioterapia no Brasil (Tabela 2) $(15,16)$.

Tabela 1 - Total de cursos de pós-graduação stricto sensu recomendados e reconhecidos pela Capes por grande área

\begin{tabular}{lccc}
\hline Grande área & Doutorados & $\begin{array}{c}\text { Mestrados } \\
\text { acadêmicos }\end{array}$ & $\begin{array}{c}\text { Mestrados } \\
\text { profissionais }\end{array}$ \\
\hline Ciências da saúde & 303 & 412 & 45 \\
\hline Ciências humanas & 215 & 394 & 7 \\
Ciências agrárias & 188 & 299 & 14 \\
Ciências biológicas & 170 & 222 & 10 \\
Ciências exatas e & 169 & 256 & 11 \\
da terra & & & \\
Engenharias & 147 & 276 & 49 \\
Ciências sociais & 131 & 316 & 52 \\
aplicadas & & & \\
Multidisciplinar & 105 & 234 & 84 \\
Linguística, letras & 85 & 162 & 0 \\
e artes & & & \\
\hline
\end{tabular}

Fonte: Ministério da Educação (16).

Tabela 2 - Total de cursos de pós-graduação stricto sensu recomendados e reconhecidos pela Capes por grande área e áreas de avaliação

\begin{tabular}{lccc}
\hline Área de avaliação & Doutorado & $\begin{array}{c}\text { Mestrado } \\
\text { acadêmico }\end{array}$ & $\begin{array}{c}\text { Mestrado } \\
\text { profissional }\end{array}$ \\
\hline Medicina I & 63 & 69 & 2 \\
Medicina II & 62 & 70 & 1 \\
Odontologia & 48 & 72 & 18 \\
Medicina III & 41 & 39 & 0 \\
Saúde coletiva & 22 & 37 & 16 \\
Farmácia & 21 & 34 & 4 \\
Enfermagem & 20 & 35 & 3 \\
Educação física & 11 & 22 & 0 \\
Nutrição & 8 & 17 & 0 \\
Fonoaudiologia & 5 & 7 & 1 \\
\hline Fisioterapia/ & 2 & 10 & 0 \\
terapia ocupacional & & & \\
\hline
\end{tabular}

Fonte: Ministério da Educação (16).
Há hoje aproximadamente 28.111 doutores e 43.060 mestres na grande área ciências da saúde. Desses, 1.145 são fisioterapeutas/TO com curso de doutorado e 4.675 são fisioterapeutas/TO com curso de mestrado (17). Isso representa $4 \%$ e $11 \%$, respectivamente, dos profissionais stricto sensu da grande área da saúde e $1 \%$ e $6 \%$, respectivamente, dos profissionais de fisioterapia do Brasil, se for considerada a quantidade de fisioterapeutas até 2004. Dentro das profissões de saúde, a fisioterapia ocupa a penúltima posição em quantidade de doutores. A medicina, no topo da classificação, tem 13 vezes mais doutores que a fisioterapia (Tabela 3).

A taxa de doutores em Fisioterapia em relação aos de Medicina é de 1 para 13 (Tabela 1), enquanto a taxa de cursos de doutorado em Fisioterapia em relação aos de Medicina é de 1 para 83 (Tabela 2). Ao se comparar as duas taxas, era de se esperar uma quantidade bem maior de doutores em Medicina em relação à Fisioterapia. Pode-se deduzir que ou as vagas nos cursos de doutorado em Fisioterapia são bem maiores que nos de Medicina ou os fisioterapeutas estão buscando com mais intensidade os cursos de doutorado.

Dentro desse contexto a Fisioterapia, com sua pós-graduação stricto sensu, passou a fazer parte de um rígido processo anual e trienal de avaliação da Capes, o qual segue regras bem estabelecidas pela

Tabela 3 - Número de doutores e mestres nas profissões na grande área ciências da saúde

\begin{tabular}{lcccc}
\hline & Doutores & Mestres & \%* & \%** \\
\hline $\begin{array}{l}\text { Grande área } \\
\text { ciências da saúde }\end{array}$ & 28.111 & 43.060 & & \\
Medicina & 14.435 & 13.677 & $51 \%$ & $32 \%$ \\
Odontologia & 4.268 & 5.738 & $15 \%$ & $13 \%$ \\
Farmácia & 2.644 & 4.401 & $9 \%$ & $10 \%$ \\
Enfermagem & 1.915 & 5.143 & $7 \%$ & $12 \%$ \\
Educação física & 1.555 & 4.929 & $6 \%$ & $11 \%$ \\
Nutrição & 1.533 & 2.967 & $5 \%$ & $7 \%$ \\
\hline Fisioterapia e & 1.145 & 4.675 & $4 \%$ & $11 \%$ \\
terapia ocupacional & & & & \\
\hline Fonoaudiologia & 616 & 1.530 & $2 \%$ & $4 \%$ \\
\hline
\end{tabular}

Legenda: * $=\%$ em relação à quantidade de doutores de ciências da saúde; ${ }^{* *}=\%$ em relação à quantidade de mestres de ciências da saúde.

Fonte: Plataforma Lattes (17). 
comunidade científica mundial. Essas avaliações pontuam os cursos existentes de forma a interromper seu funcionamento, se for de insuficiente competência, ou de classificá-los com bons conceitos, se tiverem os devidos merecimentos qualitativos (9).

Os cursos de graduação em Fisioterapia, atualmente, possuem também um processo de avaliação de gravidade. 0 Instituto Nacional de Estudos e Pesquisa Educacionais Anísio Teixeira (Inep) conduz todo o Sistema Nacional de Avaliação do Ensino Superior no país (Sinaes), e na graduação os instrumentos que subsidiam a produção de indicadores de qualidade e os processos de avaliação de cursos são o Exame Nacional de Desempenho de Estudantes (Enade), a autoavaliação da instituição de ensino superior (IES) e a avaliação in loco realizada pelas comissões de especialistas (18).

0 Enade tem como objetivo aferir o rendimento dos alunos dos cursos de graduação em relação aos conteúdos programáticos, suas habilidades e competências. Quando a nota do exame é igual ou menor a dois, a instituição avaliada receberá a visita da comissão de especialistas para uma avaliação in loco (18). Diferentemente disso, as avaliações dos cursos de pós-graduação lato sensu em Fisioterapia possuem baixa ou nenhuma avaliação (9).

No Brasil, existem atualmente dez programas de pós-graduação stricto sensu na área de fisioterapia e terapia ocupacional que oferecem mestrados acadêmicos e apenas dois oferecem também doutorado.
Desses programas, cinco estão no Estado de São Paulo, um no Rio de Janeiro, um em Minas Gerais, um no Paraná e dois na Região Nordeste, um no Rio Grande do Norte e um em Pernambuco (Quadro 1) (19).

\section{Produção científica da fisioterapia}

O SCImago Journal and Country Rank é um portal, que inclui os fatores de impacto de revistas e países, desenvolvido com informações contidas na base de dados Scopus desde 1996 e cobre 35\% dos periódicos científicos do mundo (20). Esses indicadores podem ser usados para analisar a produção científica desses países e dessas revistas (21). Para mostrar a validade dos fatores de impacto, Saha, Saint e Christakis conduziram um estudo associando a classificação de nove revistas, segundo os fatores de impacto, à classificação destas dada por um grupo de 113 médicos da associação médica americana e a de outro grupo de 151 pós-doutores na área de saúde. 0 resultado encontrado foi de $r^{2}=0,82$ e $p=0,001$ e $r^{2}=0,83$ e $p=0,001$ para o primeiro e o segundo grupo, respectivamente (22).

Considerando a produção científica em fisioterapia, de acordo com o ranking do SCImago, levando em conta o período de 1996 a 2008, o Brasil encontra-se em diferentes posições nesse ranking, dependendo do quesito em questão. Sua melhor colocação em relação ao mundo é quando se leva em conta o

Quadro 1 - Relação de cursos de pós-graduação stricto sensu em Fisioterapia - recomendados e reconhecidos pela CAPES -, instituições de ensino superior (IES), unidade de federação (UF) e conceito

\begin{tabular}{lcccc}
\hline Programa & IES & UF & $\begin{array}{c}\text { Conceito } \\
\text { doutorado }\end{array}$ & $\begin{array}{c}\text { Conceito } \\
\text { mestrado acadêmico }\end{array}$ \\
\hline Fisioterapia & UFSCar & SP & 5 & 5 \\
Ciências da reabilitação & UFMG & MG & 5 & 5 \\
Fisioterapia & Unimep & SP & & 4 \\
Ciências da reabilitação & Unisuam & RJ & & 3 \\
Ciências da reabilitação & UEL & PR & & 3 \\
Ciências da reabilitação & Uninove & SP & & 3 \\
Fisioterapia & UFPE & PE & & 3 \\
Fisioterapia & UFRN & RN & & 3 \\
Fisioterapia & Unesp & SP & & 3 \\
Fisioterapia & Unicid & SP & & 3 \\
\hline
\end{tabular}

Fonte: Ministério da Educação (19). 
número total de documentos produzidos (11ํㅣgar). No entanto, essa colocação piora (30ํ lugar) quando se considera o índice $\mathrm{H}$, parâmetro que indica a qualidade dos pesquisadores, baseado no número de artigos publicados em um período e o número de citações de cada artigo (21). Dentre os países da América Latina, o Brasil encontra-se em 1 o lugar na maioria dos quesitos, piorando essa colocação também no índice $\mathrm{H}$ ( $2^{\circ}$ lugar) e no número de citações por documento ( $5^{\circ}$ lugar), fator que influencia o índice H (Tabela 4).

Quando observados separadamente os anos de 2008 e 2003, a produção científica do Brasil em fisioterapia melhorou muito sua colocação no mundo (de 25ำ para 4ํㅜ colocado) se considerado o número total de documentos. Contudo, quando avaliada segundo o índice $\mathrm{H}$, a produção cientifica brasileira na área de fisioterapia quase não muda entre os anos de 2003 e 2008 (Tabela 5). Em relação à América Latina, o Brasil manteve sua boa colocação, não havendo nenhuma alteração relevante entre os anos de 2003 e 2005 (Tabela 6).

\section{Divulgação científica em fisioterapia}

As revistas de fisioterapia constituem o meio de comunicação mais usado para a divulgação dos conhecimentos produzidos pela pesquisa, representando a nossa cultura científica e o nosso patrimônio intelectual. Por meios delas, pode-se fazer uma análise do progresso científico, pois a produção científica possui estreita relação com os periódicos, no que se refere à qualidade e à quantidade, uma vez que esse é o meio de veiculação das pesquisas. 0 número e a qualidade das revistas de uma determinada área ou de um determinado país são dados importantes no processo de avaliação dessa produção (23).

Existem atualmente 12 revistas brasileiras de fisioterapia, sendo as mais antigas a Fisioterapia em Movimento, fundada em 1989, hoje com conceito Qualis B2 (Quadro 2). Em seguida, a Acta Fisiátrica e Fisioterapia e Pesquisa, de 1994, hoje com mesmo conceito Qualis da Fisioterapia em Movimento. Essa quantidade parece ser insuficiente para atender à demanda de publicação dos conhecimentos científicos

Tabela 4 - Colocação da produção científica do Brasil em fisioterapia, em relação ao mundo e à América Latina

\begin{tabular}{lcccccc}
\hline & Índice H & $\begin{array}{c}\text { Número de } \\
\text { documentos }\end{array}$ & $\begin{array}{c}\text { Documentos } \\
\text { citáveis }\end{array}$ & Citações & Autocitações & $\begin{array}{c}\text { Citação por } \\
\text { documento }\end{array}$ \\
\hline Brasil no mundo & 30 & 11 & 11 & 28 & 26 & 44 \\
Brasil na América Latina & 2 & 1 & 1 & 1 & 1 & 5 \\
\hline
\end{tabular}

Fonte: SCImago Journal and Country Rank (20).

Tabela 5 - Colocação da produção científica do Brasil em fisioterapia, em relação ao mundo, nos anos de 2003 e 2008

\begin{tabular}{lcccccc}
\hline & Índice H & $\begin{array}{c}\text { Número de } \\
\text { documentos }\end{array}$ & $\begin{array}{c}\text { Documentos } \\
\text { citáveis }\end{array}$ & Citações & Autocitações & $\begin{array}{c}\text { Citação por } \\
\text { documento }\end{array}$ \\
\hline Brasil no mundo 2008 & 30 & 4 & 4 & 15 & 10 & 49 \\
Brasil no mundo 2003 & 29 & 25 & 24 & 31 & 28 & 29 \\
\hline
\end{tabular}

Fonte: SCImago Journal and Country Rank (20).

Tabela 6 - Colocação da produção científica do Brasil em fisioterapia, em relação à América Latina, nos anos de 2003 e 2008

\begin{tabular}{lcccccc}
\hline & Índice H & $\begin{array}{c}\text { Número de } \\
\text { documentos }\end{array}$ & $\begin{array}{c}\text { Documentos } \\
\text { citáveis }\end{array}$ & Citações & Autocitações & $\begin{array}{c}\text { Citação por } \\
\text { documento }\end{array}$ \\
\hline Brasil na América Latina 2008 & 2 & 1 & 1 & 1 & 1 & 3 \\
Brasil na América Latina 2003 & 2 & 1 & 1 & 2 & 2 & 2 \\
\hline
\end{tabular}

Fonte: SCImago Journal and Country Rank (20). 
gerados e formar um banco de dados que permita o ensino da fisioterapia baseada em evidências.

Isso já foi mostrado anteriormente por Madeira et al. (23), em seu estudo que se referiu ao pequeno número de periódicos da área da fisioterapia, comparando com a odontologia, que na época se encontrava com 74 revistas, contra 12 da fisioterapia. Em outro estudo, Filippin e Wagner relacionaram a fisioterapia e a medicina, referindo que a fisioterapia, ao contrário da medicina, ainda não tem pesquisas suficientes para formar um corpo científico de conhecimento necessário para sustentar a prática baseada

Quadro 2 - Relação das revistas brasileiras de fisioterapia

\begin{tabular}{|c|c|c|c|c|c|c|c|}
\hline Título & $\begin{array}{c}\text { Ano de } \\
\text { fundação }\end{array}$ & Periodicidade & ISSN & $\begin{array}{l}\text { Filiação e } \\
\text { procedência }\end{array}$ & Indexação & Qualis & Sites \\
\hline $\begin{array}{l}\text { Revista } \\
\text { Brasileira de } \\
\text { Fisioterapia }\end{array}$ & 1996 & Semestral & $1413-3553$ & $\begin{array}{l}\text { Universidade } \\
\text { Federal de São } \\
\text { Carlos }\end{array}$ & $\begin{array}{l}\text { SciEL0, } \\
\text { Scopus, } \\
\text { EmCare, Web } \\
\text { of Science, } \\
\text { ISI, MEDLINE }\end{array}$ & B1 & www.rbf-bjpt.org.br \\
\hline Acta Fisiátrica & 1994 & Trimestral & $0104-7795$ & USP & $\begin{array}{l}\text { LILACS, } \\
\text { Latindex }\end{array}$ & B2 & www.actafisiatrica.org.br \\
\hline $\begin{array}{l}\text { Fisioterapia } \\
\text { em Movimento }\end{array}$ & 1989 & Trimestral & $0103-5150$ & PUCPR & $\begin{array}{l}\text { SciELO, } \\
\text { CINAHL, } \\
\text { CNEN, IBICT, } \\
\text { Latindex, } \\
\text { LILACS, } \\
\text { Sumários.org }\end{array}$ & B2 & www.pucpr.br/revfisio \\
\hline $\begin{array}{l}\text { Fisioterapia e } \\
\text { Pesquisa }\end{array}$ & 1994 & Quadrimestral & $1809-2950$ & USP & $\begin{array}{l}\text { LILACS, } \\
\text { Latindex, } \\
\text { CINAHL }\end{array}$ & B2 & www.revistasusp.sibi.usp.br \\
\hline $\begin{array}{l}\text { Fisioterapia } \\
\text { Brasil }\end{array}$ & 2000 & Bimestral & $1518-9740$ & $\begin{array}{l}\text { Editora } \\
\text { Atlântica, RJ }\end{array}$ & LILACS & B3 & www.atlanticaeditora.com.br \\
\hline FisioBrasil & 2002 & Bimestral & $1676-1324$ & $\begin{array}{l}\text { Revista Brasileira } \\
\text { de Fisioterapia }\end{array}$ & SciELO & B5 & www.fisiobrasil.com.br \\
\hline $\begin{array}{l}\text { Revista Terapia } \\
\text { Manual }\end{array}$ & 2002 & Trimestral & $1677-5937$ & $\begin{array}{l}\text { Escola de } \\
\text { Terapia Manual } \\
\text { e Postural } \\
\text { Londrina, PR }\end{array}$ & $\begin{array}{l}\text { CINAHL, } \\
\text { SciELO, } \\
\text { LILACS }\end{array}$ & B5 & www.revistaterapiamanual.com.br \\
\hline $\begin{array}{l}\text { Fisioterapia } \\
\text { SER }\end{array}$ & 2006 & Trimestral & $1809-3469$ & Editora Ser, RJ & & B5 & www.editoraser.com.br \\
\hline NovaFisio & 1996 & Bimestral & $1676-1324$ & & & B5 & www.novafisio.com.br \\
\hline $\begin{array}{l}\text { Revista } \\
\text { Brasileira de } \\
\text { Fisioterapia } \\
\text { do Trabalho }\end{array}$ & 2010 & Trimestral & & Ibrafit & & & www.rbft.com.br \\
\hline $\begin{array}{l}\text { Revista de } \\
\text { Fisioterapia } \\
\text { da UNICID }\end{array}$ & 2000 & Semestral & $1518-2282$ & $\begin{array}{l}\text { Universidade } \\
\text { Cidade de São } \\
\text { Paulo }\end{array}$ & & & www.unicid.br \\
\hline $\begin{array}{l}\text { Revista } \\
\text { Virtual de } \\
\text { Fisioterapia }\end{array}$ & 2006 & & & & & & www.intramed.uol.com.br \\
\hline
\end{tabular}

Fonte: Dados da Pesquisa. 
em evidências (24). Um periódico científico é um veículo especializado com linguagem técnica para divulgação dos resultados de investigações científicas, sendo necessário que possua número internacional normalizado para publicações seriadas ou International Standart Serial Number (ISSN). Esse identificador é aceito internacionalmente para individualizar o título de uma publicação seriada e também base de indexação (Scopus, PubMed, SciELO, LILACS e outras)(25).

A área 21 compreende as especialidades das áreas profissionais da educação física, fisioterapia e terapia ocupacional. Os periódicos dessas áreas são estratificados de acordo com suas bases de indexação e são divididos em estratos de qualidade: A1 (estrato 7) - 100 pontos; A2 (estrato 6) - 80 pontos; B1 (estrato 5) - 60 pontos; B2 (estrato 4 ) - 40 pontos; B3 (estrato 3) - 20 pontos; B4 (estrato 2) - 10 pontos; B5 (estrato 1) - 5 pontos; e C (estrato 0) sem pontuação (Quadro 3).

Quando comparado o número de revistas de fisioterapia brasileiras no SCImago em relação a alguns outros países, o Brasil encontra-se bem atrás de países como Estados Unidos (14 revistas) e Reino Unido (9 revistas), porém, à frente da Austrália (1 revista) (Tabela 7). As revistas brasileiras da área de fisioterapia no SCImago são duas: a Revista Brasileira de Fisioterapia, incluída em 2007, e a Acta Ortopédica, incluída em 2006 (20).

0 número de revistas brasileiras da área de fisioterapia no SCImago é menor que o de revistas de outras profissões da área de saúde, e a maior diferença aparece quando se compara à medicina (14 revistas), profissão mais antiga e com maior número de programas de pós-graduação stricto sensu e, consequentemente, com maior produção científica (Tabela 8).

Tabela 7 - Quantidade de revistas de fisioterapia no SCImago de cada país

\begin{tabular}{lc}
\hline & Número de revistas no SCImago \\
\hline EUA & 14 \\
Reino Unido & 9 \\
Japão & 3 \\
Brasil & 2 \\
Austrália & 1 \\
Argentina & 0 \\
\hline
\end{tabular}

Fonte: SCImago Journal and Country Rank (20).

Tabela 8 - Quantidade de revistas brasileiras de diferentes profissões da área da saúde no SCImago

\begin{tabular}{lc}
\hline & Número de revistas no SCImago \\
\hline Medicina & 63 \\
Psicologia & 11 \\
Odontologia & 5 \\
Enfermagem & 4 \\
Fisioterapia & 2 \\
Fonoaudiologia & 1 \\
\hline
\end{tabular}

Fonte: Fonte: SCImago Journal and Country Rank (20).

Quadro 3 - Critérios Qualis Capes

\begin{tabular}{lccl}
\hline Estratos & $\begin{array}{c}\text { Indicativos } \\
\text { de qualidade }\end{array}$ & Pontuação & Bases de indexação \\
\hline 7 & A1 & 100 & JCR, Scopus \\
6 & A2 & 80 & JCR, Scopus \\
5 & B1 & 60 & MEDLINE, SciELO \\
4 & B2 & 40 & LILACS, CINAHL, Embase, ERIC, SPORTDiscus, Latindex \\
3 & B3 & 20 & LILACS, CINAHL, Embase, ERIC, SPORTDiscus, Latindex \\
2 & B4 & 10 & LILACS, CINAHL, Embase, ERIC, SPORTDiscus, Latindex \\
1 & B5 & 5 & LILACS, CINAHL, Embase, ERIC, SPORTDiscus, Latindex \\
0 & C & 0 & Physical Education Index, CAB Abstracts, Periódica, Open Journal Systems, Scientific \\
& & & Cambridge Abstracts \\
\hline
\end{tabular}

Fonte: Sampaio (25). 


\section{Conclusão}

A fisioterapia brasileira, além de ser uma profissão nova legalmente, é mais nova ainda como conhecimento científico. Há somente 14 anos ela foi inserida na comunidade científica brasileira. Entretanto, nesse pouco tempo já tomou medidas para assegurar a participação contínua no meio científico, a melhor qualificação de profissionais e, fundamentalmente, o reconhecimento da importância da profissão junto ao Estado, sociedade e pares.

Entretanto, muito ainda há para se fazer a fim de aumentar a (1) quantidade de pesquisadores, com a criação de outros programas stricto sensu com abrangência nacional na área; e a (2) quantidade e qualidade da produção científica e das revistas de divulgação do conhecimento científico no âmbito nacional e internacional.

Tudo isso se faz necessário se quisermos, mais tarde, aderir ao ensino e ao comportamento profissional da fisioterapia baseada em evidências, o que requer pesquisas suficientes para formar um banco de conhecimentos científicos na área e exige dos profissionais competências para ter autonomia na avaliação crítica dessas pesquisas científicas.

\section{Referências}

1. MEC. A trajetória dos cursos de graduação na área de saúde: 1991-2004. In: Haddad AE, editor. Brasília: Instituto Nacional de Estudos e Pesquisas Educacionais Anísio Teixeira; 2006.

2. Wilensky HL. The professionalization of everyone? In: Grusky 0, Miller G, editors. The sociology of organizations: basic studies. New York: The Free Press; 1970. p. 483-501.

3. Latour B. Les professions. In: Latour B. La science en action Paris: Éditions La Découverte; 1989. p. 236-86.

4. Rebellato JR. O objeto de trabalho em Fisioterapia e perspectivas de atuação e de ensino nesse campo profissional. São Carlos: Universidade Federal de São Carlos; 1986.

5. Barros FBM. Poliomielite, filantropia e Fisioterapia: o nascimento da profissão de fisioterapeuta no Rio de Janeiro dos anos 1950. Ciencia \& Saúde. 2008; 13(3):941-5.
6. Marques AP, Sanches ES. Origem e evolução da Fisioterapia: aspectos históricos e legais. Rev Fisioter Univ São Paulo. 1994;1(1):5-10.

7. Galvão AMF. Brasil, a fisioterapia e o tempo. FisioBrasil. 2000;38.

8. Marques AP, Peccin MS. Pesquisa em fisioterapia: a prática baseada em evidências e modelos de estudos. Fisioter Pesq. 2005;11(1):43-8.

9. Costa D. Dez anos de pós-graduação stricto sensu em Fisioterapia no Brasil: o que mudou? Rev Bras Fisioter. 2007;11(1):1-89.

10. Capes MEC. Mestrados/doutorados reconhecidos grande área ciências da saúde. 2010. [acesso em 3 jun. 2010]. Disponível em: http://www.capes.gov.br

11. Guirro RRJ, Costa D. Fóruns nacionais de pesquisa e pós-graduação stricto sensu em Fisioterapia. Rev Bras Fisioter. 2007;11(3):177-243.

12. Coury HJCG, Mancini MC. Representação da Fisioterapia e da terapia ocupacional no CNPq. Rev Bras Fisioter. 2008;12(3):4-6.

13. Andrade AFDD, Britto RR. Unindo forças seremos fortes! Rev Bras Fisioter. 2008;12(6):5-6.

14. Ferreira J. Mestrado para fisioterapeutas, 2010. [acesso em 3 maio 2010]. Disponível em: http:// www.unipe.br/blog/Fisioterapia

15. Capes MEC. Relação de cursos recomendados e reconhecidos - grande área, 2010. [acesso em 3 maio 2010]. Disponível em: http://www.capes.gov.br

16. Capes MdE. Mestrados/doutorados reconhecidos ciências da saúde, 2010. [acesso em 3 maio 2010]. Disponível em: http://www.capes.gov.br

17. CNPq. Plataforma Lattes, 2010. [acesso em 4 jun. 2010]. Disponível em: http://lattes.cnpq.br

18. Inep MdE. Avaliação dos cursos de graduação, 2010. [acesso em 13 maio 2010]. Disponível em: http:// www.inep.gov.br

19. Capes MEC. Relação dos cursos recomendados e reconhecidos - Fisioterapia e Terapia Ocupacional, 2010. [3 maio 2010]. Disponível em: http://www. capes.gov.br

20. SCImago. SCImago Journal and Country Rank, 2007. [acesso em 26 abr. 2010]. Disponível em: http:// www.scimagojr.com 
21. Hirsch JE. An index to quantify an individual's scientific research output. Proc Natl Acad Sci U S A. 2005; 102(46):16569-72.

22. Saha S, Saint S, Christakis DA. Impact factor: a valid measure of journal quality? J Med Libr Assoc. 2003; 91(1):42-6.

23. Madeira MC, Cirillo FP, Sauro EE, Monteiro LF. Revistas nacionais de Fisioterapia: aspectos quantitativos (comparação com as revistas de odontologia). Rev Fac Odontol. 2003;15(1):59-62.

24. Filipin LI, Wagner MB. Fisioterapia baseada em evidências: uma nova perspectiva. Rev Bras Fisiot. 2008; 12(5):432-3.
25. Sampaio JF. Respondendo às perguntas: afinal como funciona o Qualis CAPES? 0 que é Área 21/ Onde está BrJB nisso tudo? Bras J Biomotr. 2010;4(1):1-2.

Recebido: 23/01/2011

Received: 01/23/2011

Aprovado: 26/04/2011

Approved: 04/26/2011 